\title{
Stage IIA Appendix Carcinoma AJCC v8
}

National Cancer Institute

\section{Source}

National Cancer Institute. Stage IIA Appendix Carcinoma A/CC v8. NCI Thesaurus. Code C134122.

Stage IIA includes: T3, N0, M0. T3: Tumor invades through the muscularis propria into the subserosa or the mesoappendix. N0: No regional lymph node metastasis. M0: No distant metastasis. (AJCC 8th ed.) 